\title{
Basic Obesity Management Accreditation
}

A/Prof Goh Lee Gan

SFP202I; 47(5)

\section{OBESITY IN OUR COMMUNITY}

Obesity is a rising problem in Singapore. In 2010, the Singapore adult obesity rate was 10.8 percent (i.e., about one in nine adults), with 40 percent of Singaporeans having a BMI of more than $25 \mathrm{~kg} / \mathrm{m}^{2}$ (Tham KW, 2021). ${ }^{1}$ This was twice the prevalence of obesity in Singapore in 1992 which was 5.1 percent (Goh LG, Pang JSK, 2012). ${ }^{2}$ If the present trend of obesity remains unchecked, Singapore is projected to hit 15 percent of obese adults in 2024 (HPB, 2011). ${ }^{3}$

\section{CONVERSATION ON OBESITY AND WEIGHT MANAGEMENT}

Observational and self-report evidence suggest that less than half of patients with obesity were advised by their physicians to lose weight. Patients do believe it is the responsibility of their family physicians to initiate the conversation about weight management and only three percent were offended by such a conversation, according to an online survey conducted in 11 countries, involving 14,502 participants (Lam B, 2021). ${ }^{4}$

\section{TIMELY PARTNERSHIP AND SHARED VISION}

The partnership of College, Singapore Association for the Study of Obesity (SASO) and Novo Nordisk (NN) is timely. Our shared vision is that obesity is a challenging condition and needs to be managed as a chronic disease. Thanks are due to Dr Tham Kwang Wei (President) and Dr Benjamin Lam (Vice President), and members of the SASO team for putting the course materials on obesity management together in this edition of the Singapore Family Physician. Members of the SASO team presented their papers on the weekend of $3^{\text {rd }}$ and $4^{\text {th }}$ April in this obesity management skills course which had eight study units, case studies and discussions to share lived experiences.

Mr Praful from NN needed to be credited for shifting the needle as it were on the idea of training our family physicians on current thinking of obesity management as a disease. We morphed his idea of small cohorts of doctors to be trained to a mass training programme on Zoom as suggested by our College President A/Prof Tan Tze Lee. Mr Praful kindly agreed to that mass training format.

\section{COVERAGE OF THE BOMA COURSE}

Unit 1 - Understanding Obesity as a Chronic Disease by Dr Tham Kwang Wei \& Dr Benjamin Lam Chih Chiang. Obesity is now recognised as a chronic disease, requiring long-term monitoring and preventing related complications like diabetes, cardiovascular disease, and also stigmatisation.

Unit 2 - Practical Approach To The Patient With Obesity by Dr Amanda Lim Yuan Ling \& Dr Benjamin Lam Chih Chiang. The 5As framework (Ask, Assess, Advise, Agree, and Assist) is a practical framework for obesity assessment and counselling, and patient engagement.

Unit 3 - Understanding Matters of the Mind in Obesity by $\mathrm{Mr}$ Adrian Toh. Barriers to lifestyle intervention in adults are poor motivation, lack of time, environmental, societal and social pressures, health and physical limitations, negative thoughts and moods, socioeconomic constraints, gaps in knowledge/lack of awareness and lack of enjoyment of exercise. Cognitive behavioural therapy (CBT) is known to be effective in addressing unhealthy eating habits, lack of physical activity, and obesity.

Unit 4 - Dietary Interventions For Weight Loss by Ms Izabela Kerner. A variety of diet plans are available for use in the clinical setting. To date, studies have failed to demonstrate the superiority of one diet plan over another as patients can lose weight on any diet in short term. Longterm studies are lacking. Any dietary approach to weight loss should be individualised and consider the health status, personal preferences, and ability of the person to sustain the recommendation in the plan.

Unit 5 - Moving It For Weight Loss by Dr Ivy Lim. Weight loss should take a multi-pronged approach, including dietary control and physical activity. It is important to advise lifestyle changes to reduce sedentary behaviour by embarking on a light- to moderate intensity physical activity. Individuals should also be encouraged to replace sedentary behaviour with at least light-intensity physical activity whenever possible.

Unit 6 - Use of pharmacotherapy in obesity management by Dr Tham Kwang Wei. Pharmacotherapy plays a crucial role as adjunct to lifestyle and behavioural medication as well as to bariatric surgery. Available safe and effective approved anti-obesity medications for long-term use are orlistat, phentermine, and liraglutide. Understanding the indications, efficacy, side-effect profile of each of these medications will help to match the most suitable treatment to the patient.

Unit 7 - Intensifying treatment: bariatric surgery by Dr Shanker Pasupathy. Diet and lifestyle intervention form the basis of healthy weight management but are not effective in promoting substantial weight loss in morbidly obese individuals. Bariatric surgery appears to be the only intervention that produces profound, sustainable weight 
loss and excellent control of attendant co-morbidities, particularly type 2 diabetes mellitus.

Unit 8 - Monitoring and support for weight loss and maintenance by Dr Irene Chu. Current guidelines recommend at least 150 minutes of moderate-intensity physical activity per week to achieve modest weight loss. Resistance training may be added to promote the gain or maintenance of learn muscle mass and loss of body fat. Although physical activity is not an effective means of weight loss compared to dietary or surgical interventions, it provides numerous health benefits. Existing weight management guidelines recommend an increase in nonexercise and active leisure activities to minimise sedentary behaviour.

Case studies and discussions. The case studies and ensuing discussion by Dr Natalie Koh, Dr Suraj Kumar, Dr Tan Kok Kuan, Dr Donna Tan and Dr Lee Yingshan together with the speakers the practice dimension and lived experience on managing obese patients locally.

\section{BEYOND THIS FAMILY PRACTICE SKILLS COURSE}

Beyond this Family Practice Skills Course one can join SASO as a member and participate in more in-depth case based training courses on obesity management. For example, there is the Rooting Out Obesity Skills (ROOTS) Programme which is a comprehensive obesity professional education programme, developed by SASO, the Singapore General Hospital Obesity Centre in partnership with the World Obesity Federation brought in by Novo Nordisk. Another obesity management programme is the Obesity, Bariatric and Endocrine Societies (OBES) Annual Congress run yearly by SASO in partnership with the Obesity \& Metabolic Surgery Society of Singapore (OMSSS).

\section{REFERENCES}

I. Tham Kwang Wei. Obesity in Singapore. In: Goh LG. Family Practice Skills Course on Basic Obesity Management. College Mirror, 202I, Mar.Vol 47 No I: I4.

2. Goh LG, J Pang SK. Obesity in Singapore, Prevention and Control. Singapore Fam Physician 2012; 38(I): 8-13.

3. Health Promotion Board. Singaporeans and Obesity. HPB: Singapore, 2010.

4. Benjamin Lam. Physicians did not advise on obesity. In: Goh LG. Family Practice Skills Course on Basic Obesity Management. College Mirror, 201 I, Mar.Vol 47 No I: 14.

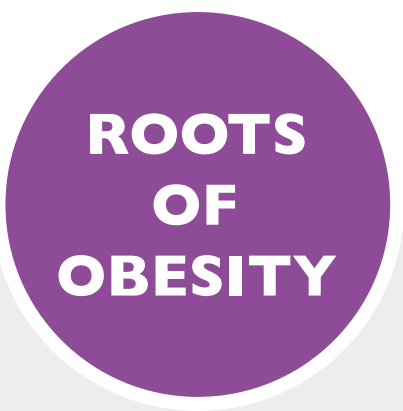

\section{Solution lies in:}

I Deep Understanding of the Roots

2 Practical Approach of 5As

3 Change Strategy

4 Dietary Intervention

5 Moving for Weight Loss

6 Adjunctive Pharmacotherapy

7 Bariatric Surgery

8 Monitoring \& Weight Loss Maintenance 